\title{
Thermal Fatigue Resistance of Bionic Compacted Graphite Cast Iron Treated with the Twice Laser Process in Water
}

\author{
Y. Liu, ${ }^{\text {a H. Zhou, }}{ }^{\text {b,1 }}$ C. Y. Yang, ${ }^{\text {c }}$ and J. Y. Cheng ${ }^{\mathrm{d}}$ \\ ${ }^{a}$ Metallurgical Engineering Department, Liaoning Institute of Science and Technology, Benxi, China \\ ${ }^{\mathrm{b}}$ Key Lab of Automobile Materials, The Ministry of Education, Jilin University, Changchun, China \\ c Jilin Liyuan Precision Manufacturing Co., Ltd., Liaoyuan, China \\ d Jinlin Automobile Brake Factory, Changchun, China \\ 1 liuyan_425@sina.com
}

For enhancing the thermal fatigue resistance of bionic compacted graphite cast iron (CGI) lasertreated in water, the bionic units are treated twice with the laser to form an uneven bionic surface imitating the alternately soft and hard structure of a plant leaf, which can resist the tearing wind. The results show that this method, without changing the phase composition of the bionic unit, makes the austenite-to-martensite transformation more complete and the content of residual austenite is reduced, in addition, the microstructure coarsens and microhardness slightly turns lower. The twice laser process in water can effectively enhance the thermal fatigue resistance of bionic CGI by reducing the number of cracks in bionic units since the number of cross cracks on the bionic unit surface is the key factor, which affects their resistance to thermal crack propagation.

Keywords: twice laser process, thermal fatigue, bionic, water, compacted graphite cast iron.

Introduction. With appearance of state-of-the-art high-speed trains, the braking properties of a compacted graphite cast iron (CGI), which has been traditionally used in brake disks, fail to meet the heavy-duty operation requirements. However, a quite lucrative direction in the development of new brake materials is the bio-inspired improvement of traditional materials based on the bionic unsmooth surface principle, which has been introduced in the studies of slidinge resistance reduction by unsmoothed bionic surface [1] and abrasive wear behavior of bamboo [2]. Zhou et al. [3,4] proposed the laser bionic treatment technique and used it to produce bionic units from the cast iron and $3 \mathrm{Cr} 2 \mathrm{~W} 8 \mathrm{~V}$ die steel with the enhanced wear and thermal fatigue resistance characteristics, due to formation of the alternate soft and hard bionic surfaces. These studies proved that the properties of bionic units are critical for improving the bionic material properties. In wear resistance tests, hardness of the bionic units is much higher than the substrate, while the wear ratio between units and frictional pairs is much lower than that of the substrate, which implies that the bionic units are equivalent to particle-reinforcement of a substrate material [5] and, thus, improve its wear resistance [5]. Under thermal fatigue conditions, the bionic units can prohibit initiation [6] and propagation [7] of thermal cracks in a substrate, enhancing the thermal fatigue resistance of materials. With further development of this approach, a laser bionic process in water medium has been reported in [8], which made it possible to improve the bionic units and achieve and the wear resistance improvement of bionic CGI by $60 \%$.

Following the above approach, this study is devoted to enhancement of thermal fatigue resistance of the bionic CGI by the twice laser treatment in water and possible brake disk application of this material.

The brake disk thermomechanical loading conditions in operation can be reduced to the following factors. During braking, a high friction force produces work, which is converted into the heat energy to be absorbed by the brake disk, so that the temperature of 
the disk working surface increases rapidly and then drops [9]. The alternate heating and cooling caused by frequent braking may lead to initiation and propagation of thermal cracks, which may cause the brake disk fracture [10]. Therefore, when improving the wear resistance of brake materials, the thermal fatigue resistance issues should not be neglected. Study [8] has shown that the laser bionic process in water can only slightly improve the thermal fatigue resistance of bionic CGI, insofar as the hardness of bionic units was increased, but their plasticity was accordingly reduced, and numerous cracks at the surfaces of bionic units appeared at the thermal fatigue test early stage. Therefore, the bionic units upon their laser treatment in water have a better resistance to thermal crack initiation, but a lower resistance to thermal crack propagation, as compared with the bionic units treated in air. For the above reason, the present study focuses on the ways to ensure a better performance of the bionic units by laser treatment in water.

In particular, the twice laser process is successfully applied to improve the performance of bionic units treated in water. Noteworthy is that the twice laser process defined here is different from the laser remelting process described elsewhere [8]. The "laser remelting process" refers to application of laser energy to achieve a partial melting-solidification of the solid metal surface, while the "twice laser process" refers to the laser energy application to melting the finished bionic unit, thus making the unit remelted and solidified. This process is also refered to as "laser overlap process" [11].

\section{Experiment.}

1.1. Materials. The test material was cut out from the brake disk of the high-speed train. Its chemical composition is listed in Table 1, while its microstructure obtained via the SEM technique using an ZEISS EVO 18 scanning electron microscope, contains vermicular graphite $(\mathrm{G})$, pearlite $(\mathrm{P})$, and ferrite $(\mathrm{F})$ as shown in Fig. 1.

$\mathrm{T}$ a $\mathrm{b} 1 \mathrm{e} 1$

\section{Chemical Composition of CGI}

\begin{tabular}{|c|c|c|c|c|c|c|c|c||}
\hline Element & $\mathrm{C}$ & $\mathrm{Si}$ & $\mathrm{Mn}$ & $\mathrm{P}$ & $\mathrm{S}$ & $\mathrm{Re}$ & $\mathrm{Mg}$ & $\mathrm{Fe}$ \\
\hline Composition (wt.\%) & 3.56 & 2.56 & 0.71 & 0.03 & 0.03 & 0.02 & 0.02 & Balance \\
\hline \hline
\end{tabular}

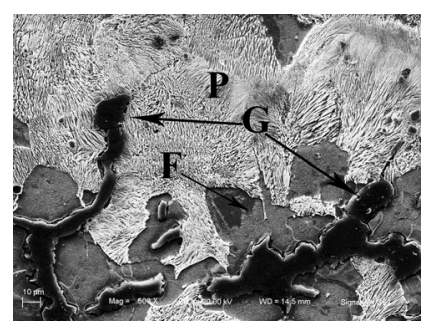

Fig. 1. The material microstructure consisting of pearlite, ferrite, and vermicular graphite.

1.2. Preparation of Bionic Specimens. The size of specimens for thermal fatigue test is $40 \times 20 \times 6 \mathrm{~mm}$. The bionic units are treated by a Nd-YAG solid state laser of $1064 \mathrm{~nm}$ wavelength and the maximum power $100 \mathrm{~W}$. The specific parameters for the laser treatment of bionic units are as follows: the single-pulse energy is $9.71 \mathrm{~J}$, pulse duration is $10 \mathrm{~ms}$, frequency is $5 \mathrm{~Hz}$, defocusing distance is $12 \mathrm{~mm}$, and the scanning speed is $0.72 \mathrm{~mm} / \mathrm{s}$. As is shown in Fig. 2a, the bionic units are parallel bands, imitating the alternately soft and hard structure of a plant leaf, which can resist the tearing wind (as shown in Fig. 2b), while the space between two units is $5.0 \pm 0.5 \mathrm{~mm}$. The tests are conducted in air and in water. The laser bionic process in water is carried out in a glass flume; the re-circulating water flow provides generation of a permanent $2 \mathrm{~mm}$-thick water film at the surface of the bionic units, 

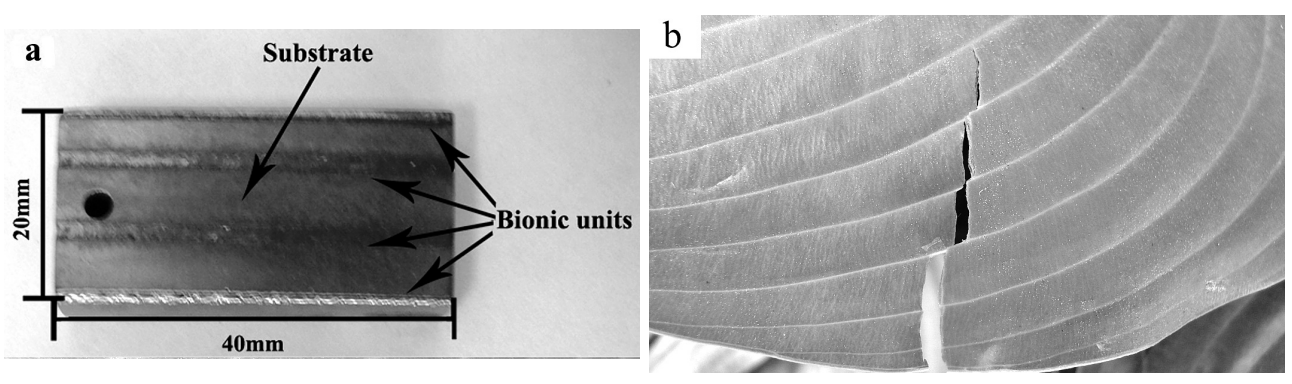

Fig. 2. The photograph of the bionic specimen and its bionic prototype: (a) photograph of the bionic specimen; (b) bionic prototype.

which ensures a higher microhardness and a larger unit area. of the laser-treated surfaces. According the earlier research [8], an increase in water film thickness results in the unit area reduction and a higher microhardness, while water film thickness of $2 \mathrm{~mm}$ provides a suitable microhardness and unit area of the bionic unit.

Hereinafter, the specimens treated in air are referred to as "in-air" specimens, those treated in water as "in-water" ones, while the specimens subjected to twice laser treatment in water as "twice-treated" ones.

1.3. Thermal Fatigue Test. Thermal fatigue test $\mathrm{s}$ are carried out using a selfrestrained thermal fatigue testing machine. The specimens are heated by a high-temperature electric resistance furnace and cooled by running water. The fixed-time gearing drive moves the specimens up and down between the furnace and water pool. The temperature at the heating period of $60 \mathrm{~s}$ is $600 \pm 5^{\circ} \mathrm{C}$, and that of the cooling period of $5 \mathrm{~s}$ is $25 \pm 5^{\circ} \mathrm{C}$. The specimens are unloaded for observation after each 100 thermal cycles at the first step (from 100 to 400 thermal cycles), and then after each 200 thermal cycles at the last step (from 800 to 1600 thermal cycles). The roll scale is removed from the specimen surface by dipping it into 3\% dilute hydrochloric acid. An Olympus PMG3 metallographic microscope is used to observe the specimen surface morphology and measure the number and length of thermal fatigue cracks.

\section{Results and Discussion.}

2.1. Phase Composition and Microstructure of Bionic Units. The phase composition of all specimens is analyzed by the X-ray diffraction (D/Max 2500 PC Rigaku, Japan), as is shown in Fig. 3. The results indicate that the phase compositions of in-water, in-air, and twice-treated specimens are similar: new phases of martensite, austenite $\left(\gamma\right.$-Fe) and $\mathrm{Fe}_{3} \mathrm{C}$ appear, in addition to the original phases of ferrite $(\alpha-\mathrm{Fe})$ and graphite. The only distinction of the twice-treated specimens is that the austenite peaks in them are smaller than in the other ones.

The SEM micrographs of the bionic unit surfaces obtained with a TSM-5500 LV scanning electron microscope) micrographs are shown in Fig. 4.

As compared with the in-air units, the microstructure of in-water units is more refined, while twice-treated specimens occupy the intermediate position by the order of microstructure sizes.

The above phenomenon can be explained as follows: the laser energy melts the initial material and turns the original phases into austenite. In the subsequent rapid cooling process, the austenite is transformed into martensite and residual austenite, while cementite formations appear around austenite [12]. Insofar as laser treatment in water provides a better cooling of the above process, it refines the microstructure of bionic units. Furthermore, when the bionic units are remelted by the laser energy, some zones of the bionic units are heated above their melting point again. Therefore, the microstructure transformation, from austenite to martensite, is more completed and the austenite content reduction occurs. In 


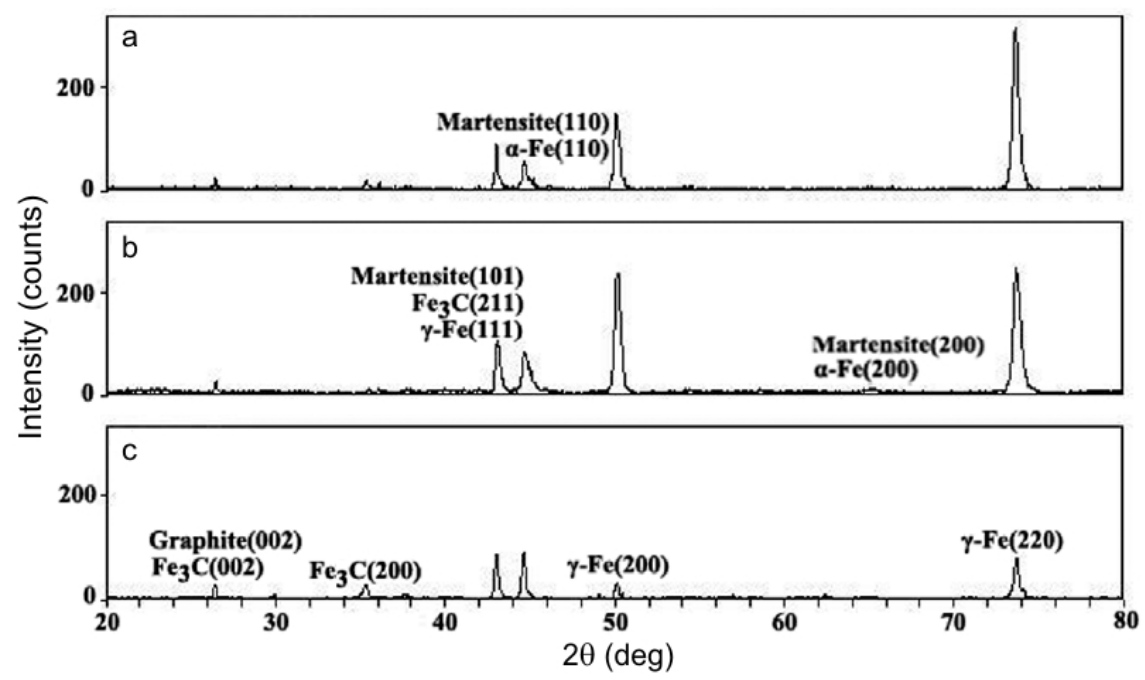

Fig. 3. XRD analysis of in-air (a), in-water (b), and twice-treated (c) bionic specimens..

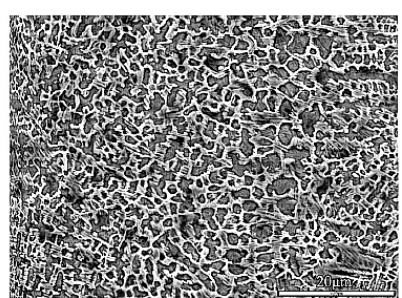

a

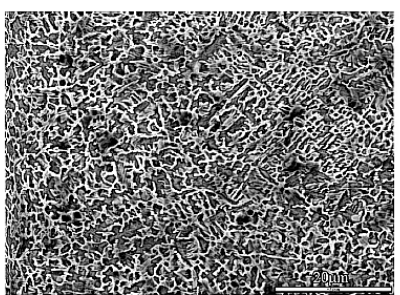

b

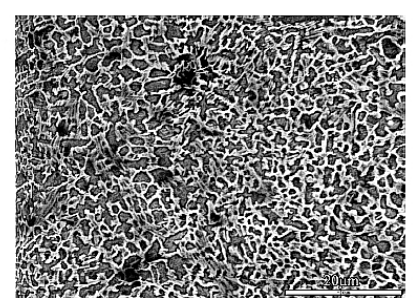

$\mathrm{c}$

Fig. 4. Microstructure of the bionic units for in-air (a), in-water (b), and twice-treated (c) specimens.

addition, due to previous laser heat action, the cooling rate decreases, and a coarser microstructure is formed.

2.2. Microhardness of Bionic Units. An 5104 Vickers microhardness tester (Buehler Co. Ltd., USA) is used to measure the microhardness of three types of bionic units with the load of $50 \mathrm{~g}$. The average values of the bionic unit surface microhardness are obtained from five masurements made in five points of each bionic unit located in the same bionic specimen. The average microhardness values of the in-air, in-water, and twice-treated bionic units are 670,920 , and $740 \mathrm{HV}$, respectively, which trend is inversly related to the order of their microstructure sizes and suggests that microstructure size of the bionic units controls their microhardness [8].

2.3. Thermal Fatigue Resistance of Bionic Specimens. The number of currently available thermal cracks longer than $0.2 \mathrm{~mm}$, as well as the length value of the longest crack is obtained by measurement data processiing software via the metallographic microscope every 200 thermal cycles during the period from 800 to 1600 thermal cycles. The respective statistical results of the number of such cracks are plotted in Fig. 5a. It can be seen that the number of thermal cracks increases with the thermal cycles for all three types of specimens. The number of cracks in the in-water and twice-treated specimens at the same loading cycles is nearly identical and always less than that for in-air specimens. The longest crack length values (as shown in Fig. 5b) also exhibit an increase with the number of thermal cycles, being arranged in the decreasing order as follows: in-water $>$ in-air $>$ twice-treated specimen crack length. 


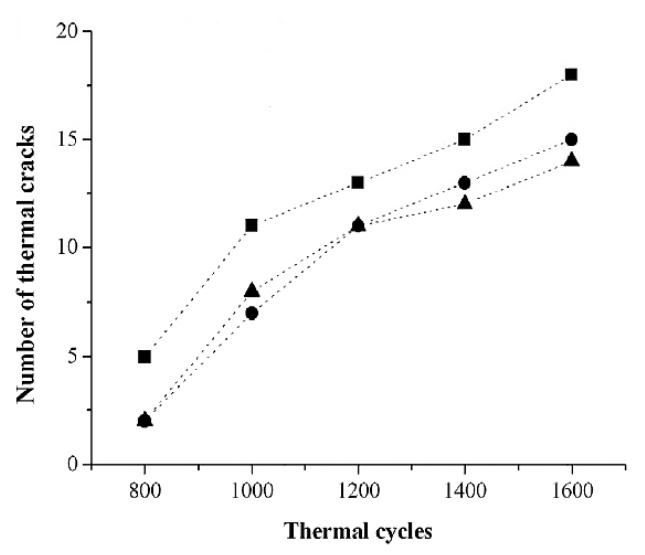

a

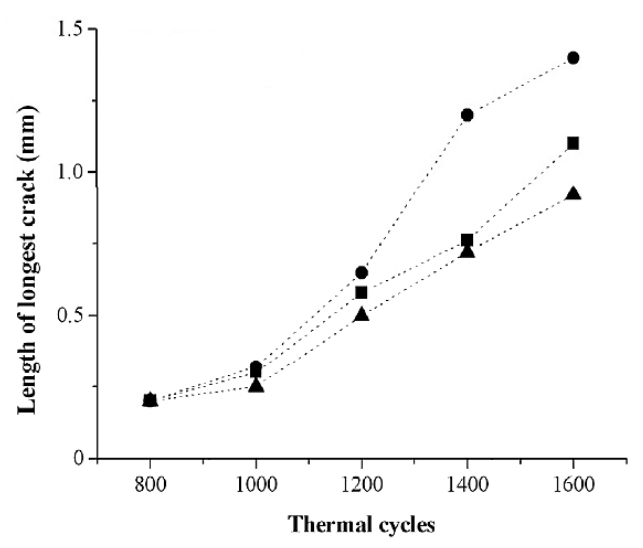

b

Fig. 5. The statistical results on the number of thermal cracks (a) and the length of the longest

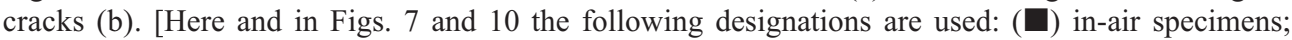
(๑) in-water specimens; ( $\boldsymbol{\Delta})$ twice-treated specimens.]

Therefore, it can be concluded that the twice laser process can enhance the thermal fatigue resistance of the bionic CGI in water.

2.4. Initial Cracks in the Bionic Units. The initial cracks were also observed at the surfaces of the bionic units before the thermal fatigue tests, as shown in Fig. 6a-c. The number of cracks obviously increases at the early period of the thermal fatigue test, as shown in Fig. 6d-f.

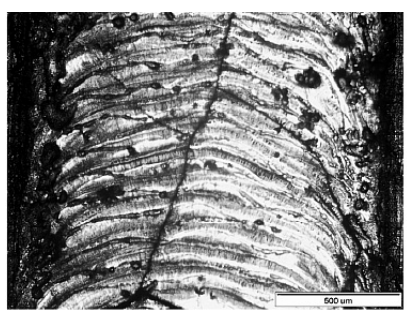

a

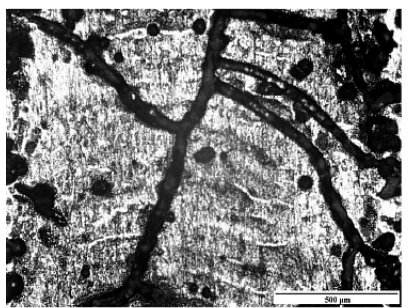

d

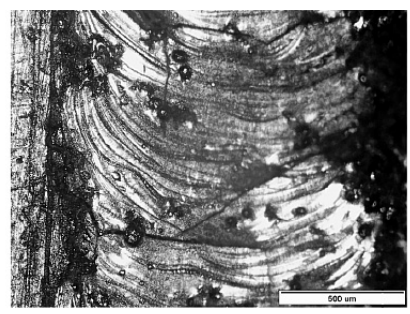

b

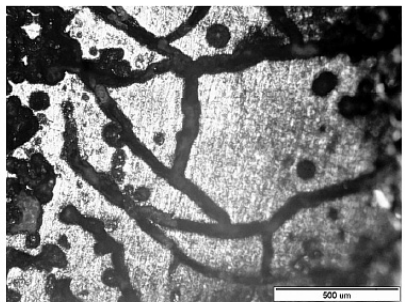

$\mathrm{e}$

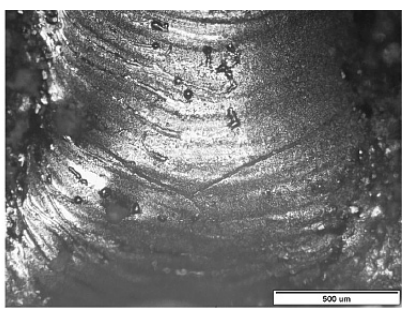

C

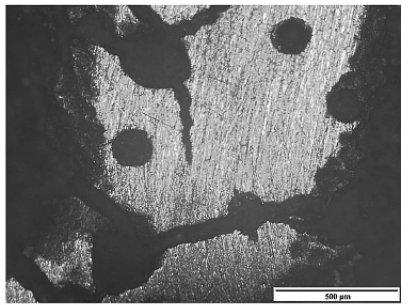

$\mathrm{f}$

Fig. 6. Cracks at the bionic unit surfaces: (a) laser-treated in air; (b) laser-treated in water; (c) lasertreated by twice laser process; (d) laser-treated in air and undergoing 400 thermal cycles; (e) lasertreated in water and undergoing 400 thermal cycles; (f) laser-treated by twice laser process and undergoing 400 thermal cycles.

The statistical results on the number of cracks at the bionic units surface are plotted in Fig. 7, taking the average measured values of four bionic units for each specimen. It can be seen that the number of cracks before the thermal fatigue test is higher for the in-air 
specimen than for the other two types. During the first 100 thermal cycles, the number of cracks in all specimens is sharply increased, while during the following 300 thermal cycles the number of cracks in the twice-treated specimens remains almost unchanged, whereas those of in-air and in-water specimens increase with the number of thermal cycles. However, further evolution of the number of cracks exhibits a sharp drop after 300 thermal cycles, which can be explained as follows: coalescence of cracks occurs with formation of a netlike texture, as shown in Fig. 6e. Therefore, in the thermal fatigue tests, the decreasing order for the number of cracks in the three types of specimens is: in-water $>$ in-air $>$ twice-treated specimen, so the respective arrangement of the bionic units by ther thermal fatigue resistance will be: twice-treated $>$ in-air $>$ in-water specimens. This implies that the twice laser process can enhance the thermal fatigue resistance of the laser-treated in water bionic units, insofar as the sources of cracks in the bionic units are microstructural defects, while the initial driving force for propagation of microcracks is the internal stress generated by the "organizational restructuring." The matrix remelting occurs to form bionic units, while remelting of bionic units improves their microstructure by healing the microstructural defects and mitigating the internal stresses, so that the number of cracks is reduced and the thermal fatigue resistance is improved.

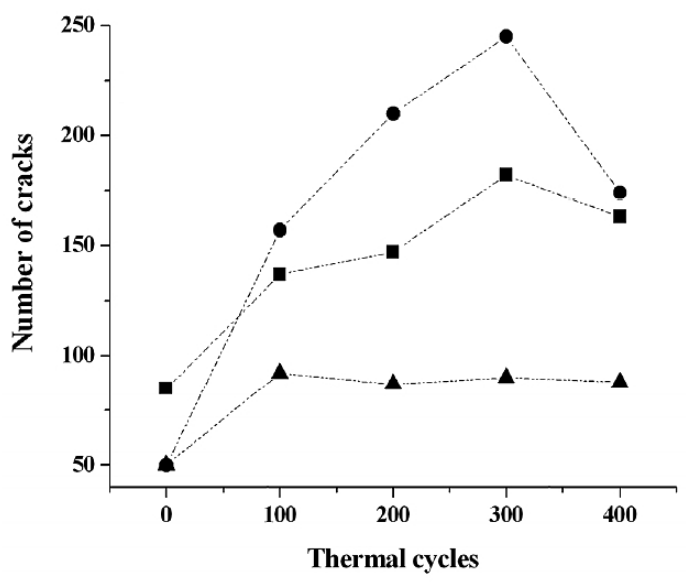

Fig. 7. Statistical results of cracks at the bionic unit surface.

\subsection{Effect of Cracks in Bionic Units on Thermal Fatigue Resistance of Bionic CGI.}

The statistical analysis of the number of thermal cracks at the specimen surfaces has revealed that cracks in the bionic units affect the measurement results on the longest crack length values. As shown in Fig. 8, when the thermal crack in the CGI substrate reaches the bionic unit border, it either stops or changes its propagation direction. But if coalescence of the thermal crack with the crack in the bionic unit occurs, their lengths will be added with the respective jumplike increase in the recorded longest crack lenth value, as is shown in Fig. 5. Thus, the number of cracks in the bionic units, which can provide a coalescence with the thermal cracks, becomes the key factor for the thermal fatigue resistance of the bionic CGI. A larger number of such cracks increases the probability of crack coalescence. In view of this, cracks in the bionic units can be classified into two types: (i) a vertical crack, which is oriented along the direction of the bionic unit and none of its two tips is located at the bionic unit edge, and (ii) a cross crack, which has at least one tip located at the bionic unit edge, as shown in Fig. 6.

It is clear that the bionic units can inhibit the thermal crack propagation if they contain no cracks or have only vertical cracks at their surface, while presence of the cross cracks increases the crack coalescence probability (Fig. 9), which should be assessed, in order to 


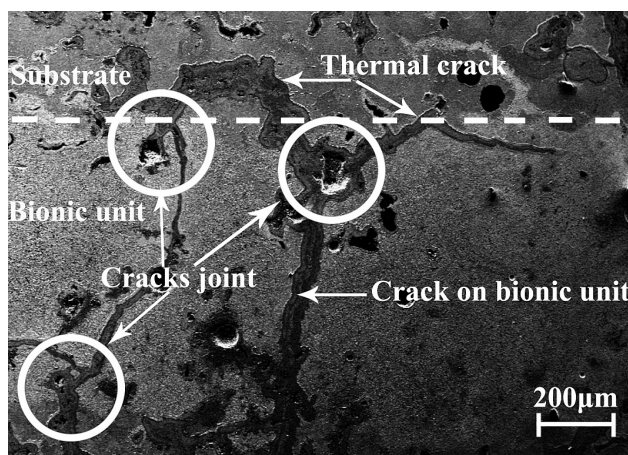

Fig. 8. Propagation of thermal crack in the CGI substrate, when the bionic unit border is reached.
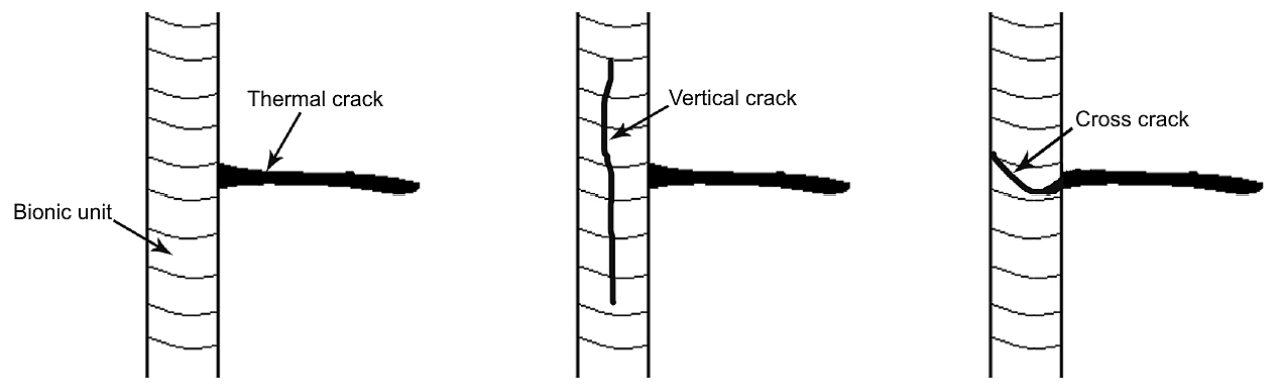

Fig. 9. Crack propagation patterns.

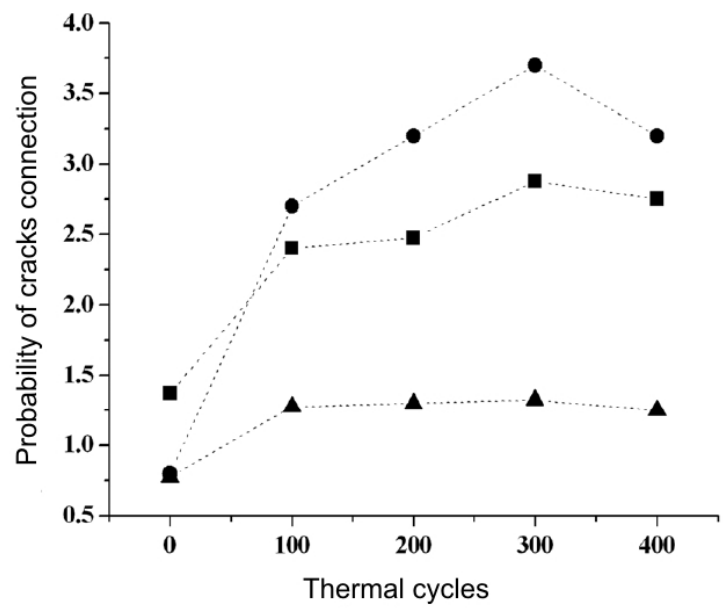

Fig. 10. Crack coalescence probability (number of cracks per unit length in $\mathrm{mm}$ ).

calculate the chance that the thermal cracks connect with the cross ones. The calculation method is quite simple: the average number of the cross cracks in the bionic units is divided by the bionic unit length. Therefore, the probability curves depicted in Fig. 10 have the patterns, which are fully identical to those shown in Fig. 7.

Conclusions. Based on the performed comparative analysis, the following conclusions can be drawn:

1. The twice laser bionic process can effectively enhance the thermal fatigue resistance of the laser-treated in water bionic CGI by reducing the number of cracks in the bionic units. 
2. The number of cross cracks at the bionic unit surface is the key factor, which controls the unit resistance to thermal crack propagation.

Acknowledgments. This article is based upon work supported by the National Science Foundation of China (Grant No. 51275200).

1. L. Q. Ren, J. Tong, and S. J. Zhang, "Reducing sliding resistance of soil against bull dozing plates by unsmoothed bionic surface," J. Terramech., 32, 303-309 (1995).

2. J. Tong, L. Q. Ren, J. Q. Li, and B. C. Chen, “Abrasive wear behaviour of bamboo," Tribology Int., 28, 323-328 (1995).

3. H. Zhou, Y. Cao, Z. H. Zhang, et al., "Thermal fatigue behavior of $3 \mathrm{Cr} 2 \mathrm{~W} 8 \mathrm{~V}$ die steel with biomimetic non-smooth surface," Mater. Sci. Eng. A, 433, 144-148 (2006).

4. H. Zhou, N. Sun, H. Y. Shan, et al., "Bio-inspired wearable characteristic surface: Wear behavior of cast iron with biomimetic units laser-treated by laser," Appl. Surf. Sci., 253, 9513-9520 (2007).

5. L. Chen, H. Zhou, Y. Zhao, et al., "Abrasive particle wear behaviors of several die steels with non-smooth surfaces," J. Mater. Process. Technol., 190, 211-216 (2007).

6. X. Tong, H. Zhou, W. Zhang, et al., "Thermal fatigue behavior of gray cast iron with striated biomimetic non-smooth surface," J. Mater. Process. Technol., 206, 473-480 (2008).

7. Z. H. Zhang, H. Zhou, L. Q. Ren, et al., "Effect of units in different sizes on thermal fatigue behavior of $3 \mathrm{Cr} 2 \mathrm{~W} 8 \mathrm{~V}$ die steel with biomimetic non-smooth surface," Int. J. Fatigue, 31, 468-475 (2009).

8. Q. C. Guo, H. Zhou, C. T. Wang, et al., "Effect of medium on friction and wear properties of compacted graphite cast iron laser-treated by biomimetic coupling laser remelting process," Appl. Surf. Sci., 255, 6266-6273 (2009).

9. J. M. Thomas, C. N. Steven, K. J. Ball, et al., "Thermal cracking in disc brakes," Eng. Fail. Anal., 9, 63-76 (2002).

10. J. Yamabe, M. Takaqi, T. Massui, and T. Kimura, "Development of disc brake rotors for strucks with high thermal fatigue strength," JSAE Rev., 23, 105-112 (2002).

11. Y. X. Li and J. Ma, "Study on overlapping in the laser cladding process," Surf. Coat. Technol., 90, 1-5 (1997).

12. K. Y. Benyounis, O. M. A. Fakron, J. H. Abboud, et al., "Surface melting of nodular cast iron by Nd-YAG laser and TIG," J. Mater. Process. Technol., 170, 127-132 (2005). 